\title{
Statistical Characteristics of Wave Breakings and their Relation with the Wind Waves' Energy Dissipation Based on the Field Measurements
}

\author{
A. E. Korinenko ${ }^{1, *}$, V. V. Malinovsky ${ }^{1}$, V. N. Kudryavtsev ${ }^{1,2}$, \\ V. A. Dulov ${ }^{1}$ \\ ${ }^{1}$ Marine Hydrophysical Institute of RAS, Sevastopol, Russian Federation \\ ${ }^{2}$ Russian State Hydrometeorological University, Saint-Petersburg, Russian Federation \\ *korinenko.alex@gmail.com
}

\begin{abstract}
Purpose. The work is aimed at studying geometric similarity of wind wave breakings in natural conditions, estimating the Duncan constant which connects the wave energy dissipation conditioned by wave breakings, with distribution of the lengths of a breaking wave fronts $\Lambda(\mathbf{c})$.

Methods and Results. The field measurements of the wave breaking characteristics were carried out at the stationary oceanographic platform located in the Golubaya Bay near the Katsiveli village. Geometric dimensions of the wave breakings' active phase, velocities and directions of their movement were determined from the video records of the sea surface; simultaneously, the meteorological information was recorded and the surface waves' characteristics were measured. Altogether 55 video recordings (duration 40-50 mins) of the sea surface were obtained. The measurements were carried out in a wide range of meteorological conditions and wave parameters (wind speed varied from 9.2 to $21.4 \mathrm{~m} / \mathrm{s}$ ).

Conclusions. It is found that the probability densities of the ratio between the maximum length of a breaking and the length of a breaking wave, obtained in various wind and wave conditions are similar. The average value of this ratio is 0.1. Distributions of the wave breakings' total length are constructed in the movement velocity intervals on a surface unit. It is shown that the experimental estimates of dependence of these distributions upon the wind speed and the wave breaking movement velocity are consistent with the theoretical predictions of O.M. Phillips (1985); at that no dependence on the waves' age was found. Quantitative characteristics of the relation between the wave lengths' distribution and the energy dissipation are obtained. The Duncan constant was estimated; it turned out to be equal to $1.8 \cdot 10^{-3}$ and independent upon the waves' and atmosphere parameters.
\end{abstract}

Keywords: wind wave breaking, energy dissipation, in situ studies, wind waves’ spectrum.

Acknowledgments: the study was carried out within the framework of the state task on theme No. 0827-2019-0003 "Fundamental studies of oceanological processes conditioning state and evolution of environment affected by the natural and anthropogenic factors, based on the observational and modeling methods". V.N. Kudryavtsev appreciates support of the Russian Scientific Foundation grant No. 17-77-30019.

For citation: Korinenko, A.E., Malinovsky, V.V., Kudryavtsev, V.N. and Dulov, V.A., 2020. Statistical Characteristics of Wave Breakings and their Relation with the Wind Waves' Energy Dissipation Based on the Field Measurements. Physical Oceanography, [e-journal] 27(5), pp. 472-488. doi:10.22449/1573-160X-2020-5-472-488

DOI: $10.22449 / 1573-160 X-2020-5-472-488$

(C) A. E. Korinenko, V. V. Malinovsky, V. N. Kudryavtsev, V. A. Dulov, 2020

(C) Physical Oceanography, 2020

\section{Introduction}

The wind waves breaking play an important role in the processes associated with the turbulence generation in the near-surface sea layer [1], gas exchange between the ocean and the atmosphere [2] and wave energy dissipation [3]. 
To determine the statistical characteristics of wind wave breakings, as a rule, video recordings of the sea surface obtained from airplanes, ships or stationary platforms are used. Based on the information determined from the video recordings (sizes and speed of breaking) [4-8], it is possible to construct the distribution $\Lambda(\mathrm{c})$ introduced by O.M. Phillips [9]. According to [9], the value $\Lambda(\mathbf{c}) d \mathbf{c}$ represents the total length of breaking fronts moving with velocities in the $(\mathbf{c}, \mathbf{c}+d \mathbf{c})$ interval on the surface unit. The proposed function $\Lambda(\mathrm{c})$ allows describing both kinematic and dynamic breaking properties. For example, the $\int \Lambda(\mathbf{c}) d \mathbf{c}$ integral is the total length of breaking fronts on the unit of the sea surface area, The sea surface fraction transferred during breaking through a given point in space per a time unit is determined by the first moment from $\Lambda(\mathbf{c})$. Higher-order moments describe the dynamic breaking properties. Energy losses due to breaking are associated with the $\Lambda(c)$ expression [9, p. 527]:

$$
S_{\text {diss }}(\mathbf{c})=b g^{-1} c^{5} \Lambda(\mathbf{c}),
$$

where $g$ is the gravitational acceleration; $b$ is the constant/proportionality parameter (the so-called Duncan constant [9]). On the assumption of the breaking similarity, O.M. Phillips considered $b$ to be a constant [9]. In [5, 10,11], $b$ was also taken as a constant. However, in laboratory studies [12-14] it was shown that $b$ is not a constant, but depends on the waves characteristics, in particular, the waves steepness. According to these measurements, the Duncan constant value varies by more than three orders ranges from от $8 \cdot 10^{-5}$ to $9 \cdot 10^{-2}$. Based on the results of [14] and using the $\Lambda$ (c) function obtained in [6], a semi-empirical model of wave energy dissipation was constructed in [15] and the $b$ dependence on the wave number of breaking waves $b(k)$ through the waves saturation spectrum $B(k)$ was found.

The complexity of the breaking process does not permit to estimate the spectral dependence $b(k)$ under natural conditions reliably. As a rule, it is assumed that $b$ is a function of the atmosphere characteristics and integral waves parameters [16-18] only and does not depend on the local scale of breaking.

The analysis of in situ measurements and the rate of turbulent energy dissipation under conditions of young wind sea is presented in [17]. The $b$ coefficient was calculated by comparing the fifth moment of $\Lambda(\mathbf{c})$ with the measured wave energy dissipation rate. According to the calculation results, no clear relation between $b$ and the wave parameters (root-mean-square slope, wave age and maximum steepness) was revealed. The average $b$ value turned out to be $3,2 \cdot 10^{-3}$

At the same time, an analysis of the available field data showed a linear dependence of $b$ on the wave age and the maximum wave steepness [18]. As noted in [18, p. 2055], if data on wave parameters is not available, it is necessary to use the average $b$ value equal to $2,1 \cdot 10^{-3}$.

At present, the issue of the $b$ parameter value and its spectral dependence remains open. Therefore, it is important to clarify the proportionality $b$ coefficient 
and determine to determine its possible changes in various hydrometeorological conditions.

The present paper is aimed to study the kinematic characteristics and geometric similarity of wind wave breakings and to estimate the Duncan constant $b$ based on the data obtained in natural conditions.

\section{Experiment}

The field studies were carried out in the autumn of 2013, 2015, 2018 and 2019 from a stationary oceanographic platform located in the Golubaya Bay near the Katsiveli village (South coast of Crimea). The oceanographic platform is installed $\sim 480 \mathrm{~m}$ from the nearest point on the coast and has coordinates $44^{\circ} 23^{\prime} 38^{\prime \prime} \mathrm{N}$., $33^{\circ} 59^{\prime} 09^{\prime \prime} \mathrm{W}$. The depth in the area of the platform is about $30 \mathrm{~m}$.

\section{Meteorological observations}

The collection of meteorological information was carried out by the multifunctional complex Davis Vantage Pro2 6152EU, located at an altitude of $23 \mathrm{~m}$ above sea level on the oceanographic platform mast The weather station facilities include a wind speed and direction meter, atmospheric pressure, humidity, air and water temperature sensors (at $3 \mathrm{~m}$ depth). The measured wind speed was recalculated into the effective neutral stratified wind speed $U$ at $10 \mathrm{~m}$ height according to the method described in [19]. In accordance with the standard procedure [20], the dynamic air velocity $u_{*}$ was calculated.

\section{Wave observations}

The characteristics of surface waves were recorded using an array of six string wave recorders located in the center and regular pentagon vertices with a circumscribed circle radius of $0.25 \mathrm{~m}$. The distance from the wave recorder trap to the nearest platform element exceeded $10 \mathrm{~m}$. Frequency-angle spectra of sea surface elevations $S(f, \phi)$ were calculated using 20-minute intervals using the maximum entropy method. The $S(f)$ frequency spectrum was determined by $S(f, \phi)$ integration according to the $\phi$ angle.

Fig. 1 shows the $S(f)$ measured during the experiments. As is known, the spectrum shape for frequencies exceeding the value of the spectral peak frequency has the form $f^{-n}$. When $n=5$, the spectrum shape corresponds to the results of [21], and when $n=4-$ to the equilibrium interval theory [9]. As can be seen from the graph, the spectra slope obtained in the present study for frequencies exceeding the spectral peak frequency is close to the law $f^{-4}$.

Field measurements, as a rule, are carried out in conditions of mixed waves, when swell waves along with wind waves appear. In this case, the swell can spread at any angle relative to the direction of the wind waves.

The approach [22] was applied to separate the frequency spectrum of waves into swell waves and waves generated by the wind. The method is based on the concept of an equilibrium interval of wind waves [9]. According to [22], the spectral domain, with its spectral level lying above the equilibrium spectrum [9], determined by the expression

$$
F(f)=2 \pi a u_{*} g(2 \pi f)^{-4},
$$


where $a=0.06$, refers to the interval of wind waves. If the spectral level lies below the curve (2), the observed waves do not belong to the class of wind waves, but are swell.

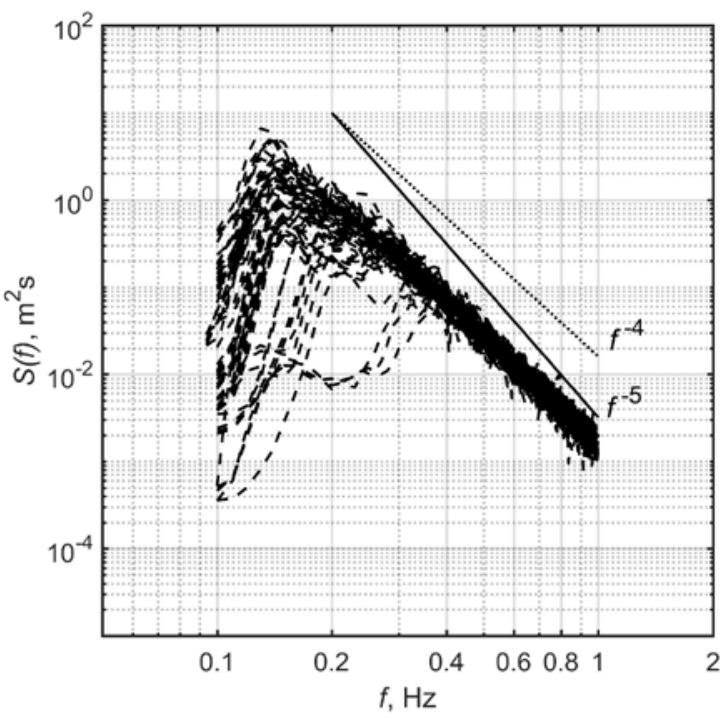

F i g. 1. Frequency spectra of the sea surface elevations (solid line shows dependence $f^{-5}$, dots dependence $f^{-4}$ )

The approach is illustrated in Fig. 2, showing the frequency spectra of surface waves obtained in our experiments, as an example. The frequency spectrum in Fig. 2, $a$ has one spectral maximum at a frequency of $f_{\mathrm{p}}=0,15 \mathrm{~Hz}$, and, according to [22], the spectral range to the right of $f_{\mathrm{p}}$ refers to wind waves. A different situation is shown in Fig. 2, $b$, demonstrating the frequency spectrum with two local maxima $f_{\mathrm{p}}=0,14 \mathrm{~Hz}$ and $f_{\mathrm{pw}}=0,28 \mathrm{~Hz}$. The spectral range lying to the right of the $f_{\mathrm{pw}}$ value refers to wind waves. The local maximum $f_{\mathrm{pw}}$ will be called the frequency of the wind wave peak. The area in the vicinity of the spectral peak at a frequency of $f_{\mathrm{p}}=0,14 \mathrm{~Hz}$ refers to swell waves, since the spectral level lies below the curve (2). Note that in the case of wind waves (Fig. 2, a), the values of the spectral peak frequency $f_{\mathrm{p}}$ and the frequency of the wind waves peak $f_{\mathrm{pw}}$ coincide.

As a result of the analysis, the values of the frequency of the spectral waves peak $f_{\mathrm{p}}$, the frequency of the wind waves peak $f_{\mathrm{pw}}$, the direction of propagation of wind waves $\phi_{\mathrm{pw}}$ and swell waves $\phi_{\mathrm{p}}$, the significant waves height $H_{\mathrm{S}}\left(H_{\mathrm{S}}=4 \sqrt{\sigma^{2}}\right.$, where $\sigma^{2}$ is the dispersion of the sea surface elevations), as well as the age of wind waves ( $\alpha=c_{\mathrm{pw}} / U$, where $c_{\mathrm{pw}}$ is the phase velocity of the waves at the frequency of the wind waves peak). 

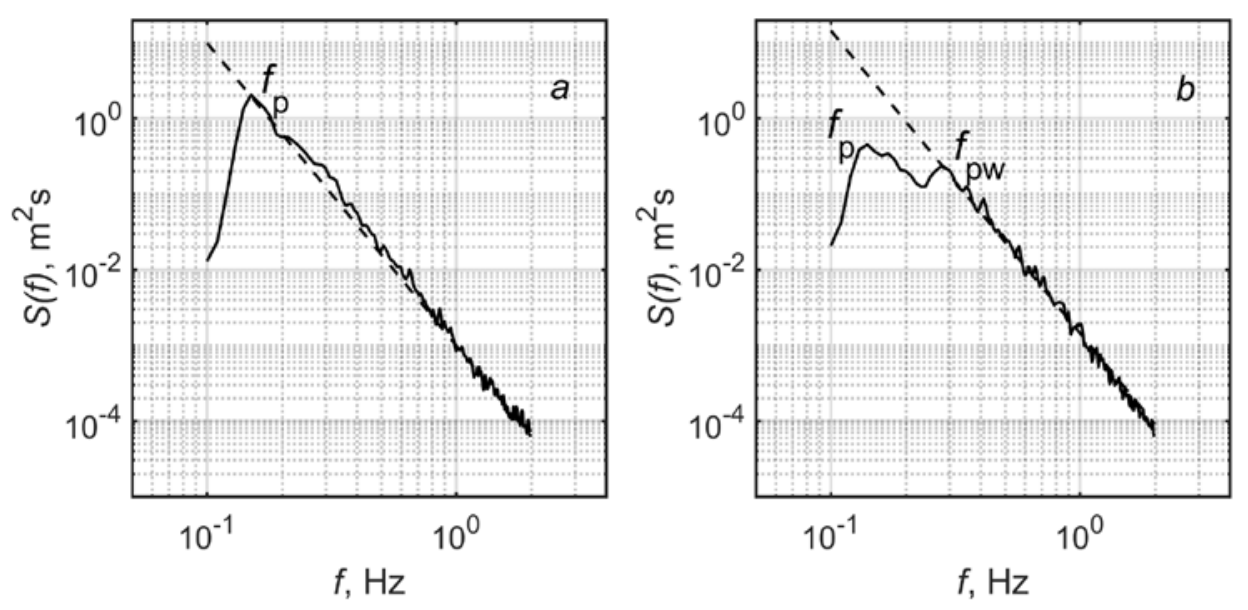

F i g. 2. Frequency spectra of the sea surface elevations: $a$ - wind waves, $u_{*}=0.55 \mathrm{~m} / \mathrm{s} ; b-$ mixed waves with the swell waves present, $u_{*}=0.71 \mathrm{~m} / \mathrm{s}$ (dash line shows dependence (2))

\section{Breakings}

The geometric characteristics of wind wave breakings were determined from video recordings of the sea surface made with a digital video camera. The camera was located at $11.4 \mathrm{~m}$ height above sea level, the sighting direction was $30^{\circ}-40^{\circ}$ to the horizon, in the azimuthal plane $-\sim 50^{\circ}-60^{\circ}$ towards the general direction of wind waves. A lens with a horizontal viewing angle of $54^{\circ}$ horizontally and $32^{\circ}$ vertically provided video recording of the site on the sea surface in the form of a trapezoid with base lengths of 14-16 m and 29-48 m. Recording was carried out at a frequency of 25 frames per second and a resolution of $1920 \times 1080$ pixels.

The breakings against the sea surface background were identified using algorithms described in [8]. With the known geometry of the survey, the image frame was tied to coordinates on a horizontal plane located at the mean sea level. Only the active phase of the breakings (breaking fronts) was distinguished, and the spreading foam spots remaining after the breaking passage were automatically filtered out. The processing result is a database of all the breaking fronts that have fallen into the camera's field of view, their characteristics, including area, coordinates, speed and propagation direction at each moment of time [8].

Fig. 3 shows an example of the temporal evolution of a single breaking area and its geometric center position.

As you can see, the braking process goes through two stages: a rapid increase in the breaking area, characterized by active generation of turbulence (blue circles) and an exponential decrease in the area (red circles). Such a time evolution of the breaking foam structure area coincides with the results of works [7, 23].

An important conclusion from the data presented in Fig. 3, b, $c$ is that wave breaking (blue circles) is moving at a constant speed. This result differs from that obtained in [5, 7], where the breaking moves with equal delay. According to the results of works [5, 7], the wave breaking velocity decreases to approximately 0.7 of the initial breaking velocity. 

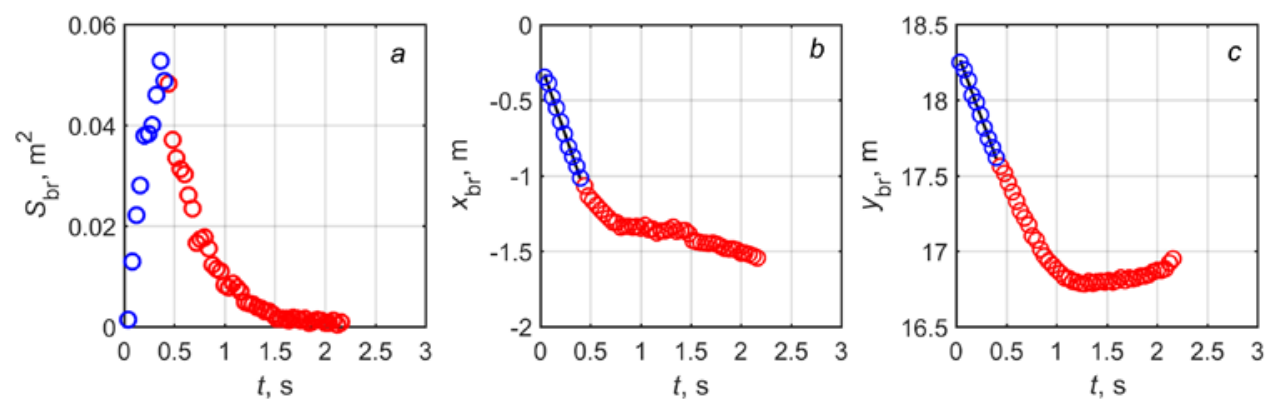

F i g. 3. Temporal evolution of wave breaking geometrical characteristics: $a$ - wave breaking squares; $b, c-x$-components and $y$-components of the wave breaking geometrical center, respectively. Blue color denotes the wave breaking parameters relating to the active phase, red color to the foam spread. Point of the coordinates' origin in Fig. 3, b, c corresponds to the point of intersection of the photolens optical axis and the sea surface

The $c$ values used in this paper were defined as follows. The components of the velocity vector $c_{\mathrm{br}}^{x}, c_{\mathrm{br}}^{y}$. were calculated from the values by the minimum error square method $x_{\mathrm{br}}(t), y_{\mathrm{br}}(t)$. The black lines in Fig. $3 b, c$ indicate linear approximations of the coordinates of the breaking geometric center. It makes possible to determine the components of the velocity vector: $x_{\mathrm{br}}(t)=c_{\mathrm{br}}^{x} \cdot t+x_{\mathrm{br}}^{0}, y_{\mathrm{br}}(t)=c_{\mathrm{br}}^{y} \cdot t+y_{\mathrm{br}}^{0}$, where $x_{\mathrm{br}}^{0}, y_{\mathrm{br}}^{0}$ are constants.

As a result, the modulus $C_{\mathrm{br}}=\sqrt{\left(C_{\mathrm{br}}^{x}\right)^{2}+\left(c_{\mathrm{br}}^{y}\right)^{2}}$ and the breaking velocity phase $\theta=\operatorname{arctg}\left(c_{\mathrm{br}}^{y} / c_{\mathrm{br}}^{x}\right)$ corresponding to its movement direction were determined.

As a result, the modulus and phase of the breaking velocity corresponding to the direction of its movement were determined.

According to [8], the breaking velocity $c_{\mathrm{br}}$ is equal to the breaking $c$ wave phase speed. However, in accordance with experimental works [13, 24, 25], the breaking velocity is proportional, but less than the phase velocity: $c_{\mathrm{br}}=\delta c$, where $\delta$ lies in the range of $0.7-0.95$. In this work, for the analysis of the breaking measurement data, $\delta=1$ will be used, then $c_{\mathrm{br}}$ is related to the $f$ wave frequency by the dispersion relation for gravitational waves: $c_{\mathrm{br}}=c=g /(2 \pi f)$.

A total 55 video recordings of the sea surface were obtained, with duration from 40 to 60 minutes each. The measurements were carried out in a wide range of meteorological conditions and wave parameters (wind speed varied from 9.2 to $21.4 \mathrm{~m} / \mathrm{s}$, wave age varied from 0.2 to 1.2 ).

\section{Results}

Dependence of the proportion of the foam-covered sea surface on the wind speed

The literature sources contain numerous measurements of the fraction of the sea surface $Q$ covered with the foam of breaking waves (see, for example, [6, 8] and the references there). Traditionally, the change in $Q$ depending on the wind speed is described by a power law, where the exponent takes on values of $\sim 2.5-5$ (see, for example, [6]). 
Fig. 4 shows the dependence of the sea surface fraction covered with breakings in the active phase on the wind speed obtained from our measurements. Each value in Fig. 4 is the result of $Q$ averaging over 20 min time interval, the solid line shows the approximation by the dependence $Q=3,5 \cdot 10^{-4} U^{2,3}$, where the coefficients are calculated by the least squares error method. Shown in Fig. 4, the wind dependence $Q$ is consistent with the results of earlier studies (see, for example, [6, 8]).

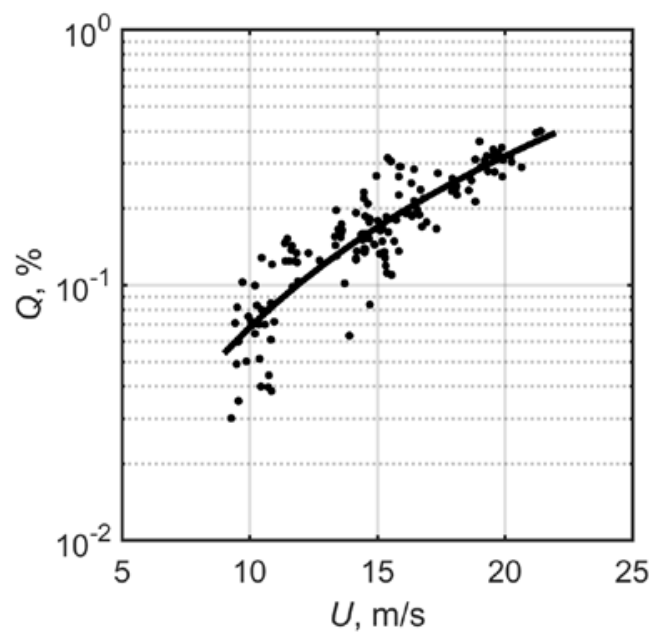

F i g. 4. Wind dependence of the sea surface part covered with breakings in the active phase

Relationship between the geometric dimensions of breaking and the breaking wave parameters

Laboratory studies [26] showed that the breakings are geometrically similar and the dimensions of breakings (in particular, the length) is proportional to the breaking wave length. The field data on the ratio $\gamma=L / \lambda$ under various hydrometeorological conditions, where $L$ is the length of the breaking fronts; $\lambda=2 \pi g^{-1} c^{2}$ is the breaking wave length is presented below.

For each video recording, from the obtained arrays $\gamma$, the histograms $\mathrm{H}(\gamma)$ were calculated and the distribution $p(\gamma)=\sum_{i=1}^{N} H(\gamma) /(N \Delta \gamma)$ where $\mathrm{N}$ and $\Delta \gamma$ are the number of samples and the interval of the histogram, respectively, was constructed. Thus, $p(\gamma)$ satisfies the normalization conditions within the limits of integration from the minimum to the maximum possible value of argument $\int p(\gamma) d \gamma=1$ and can be considered as the probability density of the random variable $\gamma$.

In Fig. 5 symbols “॰” indicate $p(\gamma)$ for each of 55 videos made at wind velocities from 9.2 to $21.4 \mathrm{~m} / \mathrm{s}$ and $\alpha$ values from 0.2 to 1.2. As follows from Fig. 5, $p(\gamma)$ values obtained in a wide range of wind velocities and wave development have a relatively small scatter. The maxima of all distributions are localized in the vicinity of the value $\gamma=0.04$. The average of $\bar{\gamma}=\int \gamma p(\gamma) d \gamma$ is $\bar{\gamma}=0,1 \pm 0,03$. 


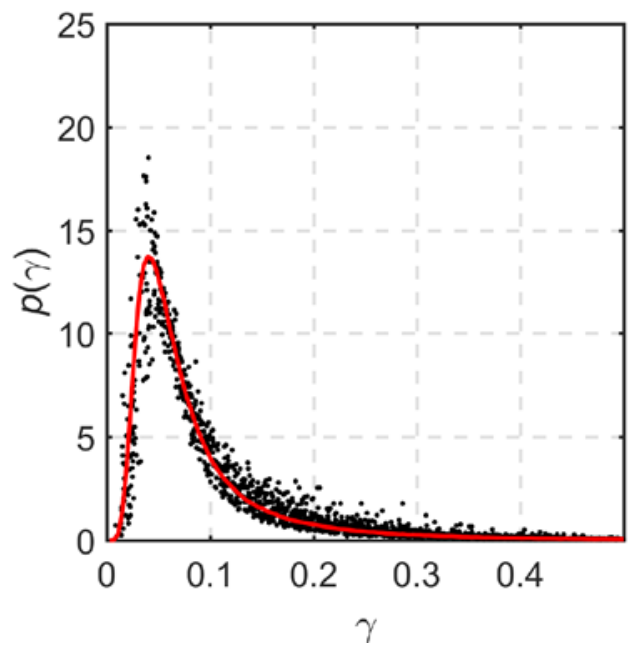

F i g. 5. Probability densities of the dimensionless values $\gamma$ (solid red line shows the result of averaging $\bar{p}(\gamma)$ of all the distributions $p(\gamma)$ with the standardization condition taken into account)

Taking into account the fact that $p(\gamma)$ distributions obtained at different $U$ and wave ages are similar and the main part of $\gamma$ values with a probability of 0.8 lies within the range $0.04 \leq \gamma \leq 0.2$, we can talk about the geometric similarity of breaking in the open sea.

In [5], the distributions $P_{\mathrm{G}}(\gamma)$ obtained at wind velocities from 11.8 to $13 \mathrm{~m} / \mathrm{s}$, both at developing and developed waves, are given. The main group of $\gamma$ values in [5] is less than 0.4, the maxima lie in the vicinity of $\gamma \approx 0.05$ value, and the distributions on the right from the maxima fall off sharply. Note that the shape of $P_{\mathrm{G}}(\gamma)$ distribution is close to that obtained in our work.

Distribution of breaking lengths

O. M. Phillips [9] proposed to use the distribution of the breaking wave fronts lengths $\Lambda(\mathbf{c})$ as a statistical measure of wave breaking. $\Lambda(\mathbf{c}) d \mathbf{c}$ represents the total length of breaking fronts per unit surface, moving in the velocity interval $(\mathbf{c}, \mathbf{c}+d \mathbf{c})$. In the equilibrium spectral range, one-dimensional distribution $\Lambda(c)$ has the form [9]

$$
\Lambda(c) \sim u_{*}^{3} c^{-6} .
$$

The one-dimensional $\Lambda(c)$ distribution according to our measurements was estimated as follows:

$$
\Lambda(c)=\frac{1}{S \cdot d c \cdot N_{\mathrm{fr}}} \sum_{k} L_{k} \mid c_{k} \in[c, c+d c],
$$

where $S$ is viewing area on the sea surface; $d c$ is the velocity interval, in our case equal to $0.5 \mathrm{~m} / \mathrm{s} ; N_{\text {fr }}$ is a number of video frames; $L_{k}$ is the length of the $k^{\text {th }}$ crest of the breaking wave moving with $c_{k}$ velocity within the interval $c_{k} \in(c, c+d c)$. PHYSICAL OCEANOGRAPHY VOL. 27 ISS. 5 (2020) 
The duration of the video recordings used to calculate $\Lambda(c)$ varied from 40 to 60 minutes.

$\Lambda(c)$ measured in this work and presented in the literature are given in Fig. 6. A feature of the displayed data is the presence of $\Lambda(c)$ maximum. For optical methods of breaking registration, $\Lambda(c)$ maximum value is observed for $2-3 \mathrm{~m} / \mathrm{s}$ breaking velocities. When using an infrared camera, $\Lambda(c)$ spectral maximum is observed when the breaking velocity is $0.8 \mathrm{~m} / \mathrm{s}$ or less. Such $\Lambda(c)$ feature is explained in detail in [28] and is associated with the fact that when using optical equipment (registering the whitecaps), the breaking of short waves (without capturing air that forms the whitecaps), which makes the main contribution to the number of breakings per unit of surface, are not identified during video processing. Thus, $\Lambda(c)$ graphs we have constructed are not statistically sufficient at $c<\sim 2,5 \mathrm{~m} / \mathrm{s}$, respectively, further we will consider the characteristics of those breakings the velocity of which exceeds $2.5 \mathrm{~m} / \mathrm{s}$. As can be seen from the figure, for the velocities of the whitecaps that exceed the velocity corresponding to $\Lambda(c)$ maximum, the functional dependence on $c$ is in good agreement with law (3). Note that the scatter of values at a fixed breaking velocity can reach two orders of magnitude.

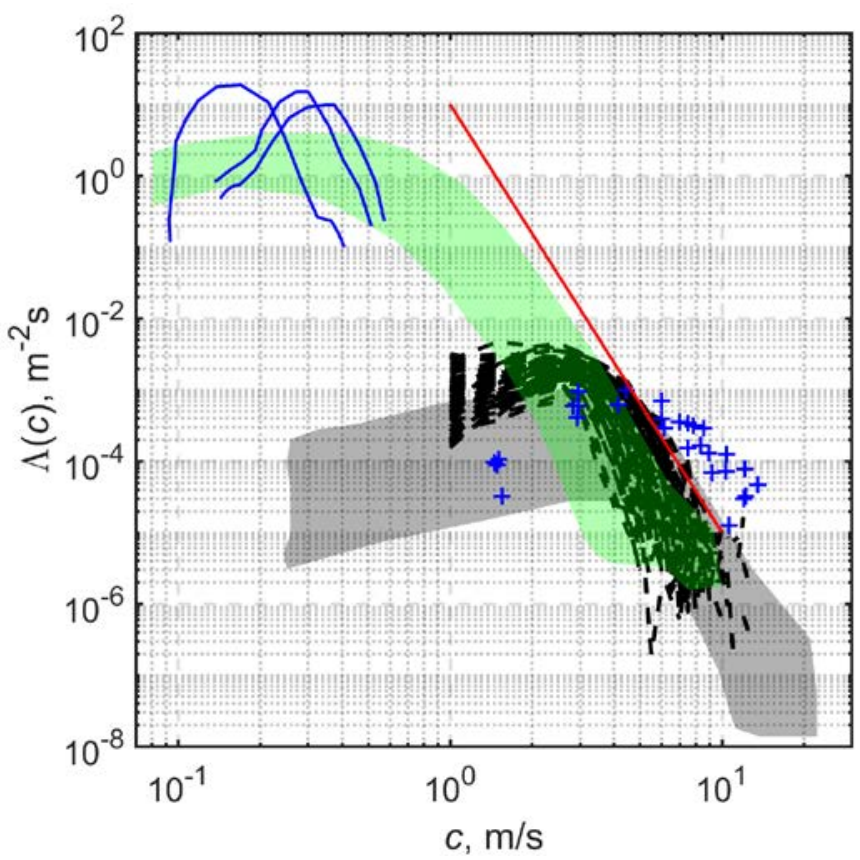

F i g. 6. Measured $\Lambda(c)$ (black dash lines) as compared to the data from [5] (blue crosses), laboratory measurements from [27] (blue lines), optical measurements data from [6] (grey area) and infrared camera measurements from [28] (green area). Red straight line denotes dependence $\sim c^{-6}$ 
$\Lambda(c)$ distributions averaged in three ranges of dynamic velocity in the air are shown in Fig. 7, $a$. At a fixed breaking velocity, $\Lambda(c)$ value increases by more than an order of magnitude with $u_{*}$ growth. In fig. 7, $b, c, \Lambda(c)$ normalized to $u_{*}^{3}$ and averaged over the dynamic velocity intervals (Fig. $7, b$ ) and over the wave age intervals (Fig. 7, $c$ ) are shown. The data in Fig. 7, $b$ after normalization to $u_{*}^{3}$ are grouped into a universal curve independent from $u_{*} . \Lambda(c) / u_{*}^{3}$ distributions averaged over the intervals of wave ages demonstrate similar behavior (Fig. 7, c). This fact indicates that there is no $\Lambda(c) / u_{*}^{3}$ dependence on the age of the waves in our database.
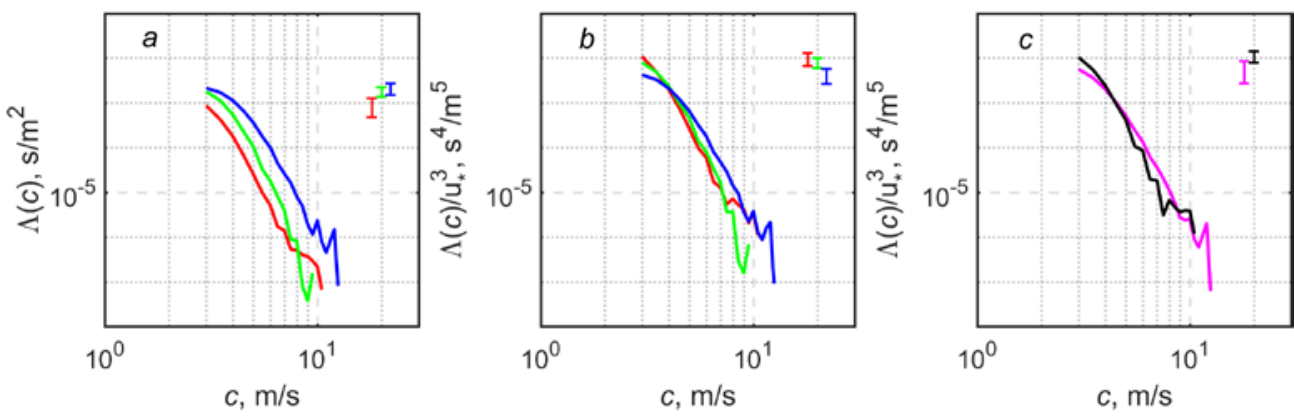

F i g. 7. Lambda of distribution (a); dependence $\Lambda(c) / u_{*}^{3}$ averaged in different ranges $u_{*}(b)$ and $\alpha(c)$ (red line - the data averaged in the range of values $u_{*}$ from 0.34 to $0.52 \mathrm{~m} / \mathrm{s}$; green line - from 0.53 to $0.67 \mathrm{~m} / \mathrm{s}$; blue line - from 0.68 to $1.00 \mathrm{~m} / \mathrm{s}$; black line - the data averaged in the range of values $\alpha$ from 0.6 to 1.1 , pink line - from 0.2 to 0.55 ; vertical sections - mean square deviations in the corresponding ranges $u_{*}$ and $\alpha$ )

Thus, the analysis of the data demonstrates that $\Lambda(c)$ have a functional dependence on $u_{*}$ and $c$ predicted by formula (3), while $\Lambda(c)$ dependence on the wave age is not clearly manifested.

Relationship between the breaking length and energy dissipation

The theory of O.M. Phillips [9] connects the kinematic characteristics of $\Lambda(\mathbf{c})$ whitecaps with the average energy dissipation rate $S_{\text {diss }}(\mathbf{c})$ due to breakings per unit surface using formula (1). According to the works [12-14], the value of Duncan's constant $b$ lies within wide limits from $8 \cdot 10^{-5}$ to $9 \cdot 10^{-2}$. At present, the question of $b$ value and its spectral dependence remains open. If, following the works $[5,10$, 11 ], we assume that $b$ is a constant value, then, according to expression (1),

$$
b=\frac{\int S_{\text {diss }}(\mathbf{c}) d \mathbf{c}}{g^{-1} \int c^{5} \Lambda(\mathbf{c}) d \mathbf{c}} .
$$


The estimation of coefficient $b$ by formula (4) was carried out within the framework of the concept of an equilibrium spectral interval [9], which assumes a local energy balance between the energy inflow from the wind and dissipation caused by breakings. According to [9], for developed wind waves, the equilibrium range of the spectrum lies within $k \in\left(2 k_{\mathrm{p}}, g u_{*}^{-2}\right)$ range, where $k_{\mathrm{p}}$ is the wave number of the spectral peak. In this work, the lower boundary of the equilibrium interval is defined as $2 k_{\mathrm{pw}}$, where $k_{\mathrm{pw}}$ is the wave number of the peak of wind waves. The upper limit is chosen equal to $k_{\mathrm{m}}=1.6 \mathrm{rad} / \mathrm{m}$, which corresponds to the minimum breaking velocity $c_{\mathrm{m}}=2.5 \mathrm{~m} / \mathrm{s}$, reliably recorded by an optical video camera.

The balance of energy inflow from wind and dissipation within the equilibrium interval is written down as

$$
\int_{2 k_{\mathrm{pw}}}^{k_{\mathrm{m}}} S_{\text {diss }}(\mathbf{k}) d \mathbf{k}=\int_{2 k_{\mathrm{pw}}}^{k_{\mathrm{m}}} S_{\text {in }}(\mathbf{k}) d \mathbf{k} .
$$

The correctness of expression (5) is confirmed by the results of [29], where it is shown that in the spectrum equilibrium range a balance of the total inflow of energy from the wind and the total dissipation of wave energy is observed.

Energy flux from the wind is equal to

$$
S_{\text {in }}(\mathbf{k})=g \omega \beta(k, \phi) F(\mathbf{k}),
$$

where $\beta(k, \phi)=0,03\left(u_{*} / c\right)^{2} \cos ^{2} \phi$ is the coefficient of waves and wind interaction [30] with an angular dependence in the form proposed in [31]; $F(\mathbf{k})$ is a spectrum of the sea surface elevation. Spatial-angular spectrum $F(k, \phi)$ was calculated by the measured frequency-angular spectrum according to the formula $F(k, \phi) k=S(\omega, \phi) \frac{d \omega}{d k}$.

Thus, expression (4) with regard to (5) will be written down as

$$
b=\frac{\int_{2 k_{\mathrm{pw}}}^{k_{\mathrm{m}}} k d k \oint S_{\mathrm{in}}(k) d \phi}{g^{-1} \int_{c_{\mathrm{m}}}^{0,7 c_{\mathrm{pw}}} c^{5} \Lambda(c) d c} .
$$

In Fig. 8 the integral dissipation rate (the denominator in expression (7)) and the integral energy inflow from the wind (the numerator in (7)) are compared. As can be seen, there is a high correlation of two independently obtained data arrays. From the data shown in Fig. 8, it follows the least-square estimates of $b=1,8 \cdot 10^{-3} \pm 4,5 \cdot 10^{-4}$ coefficient. 


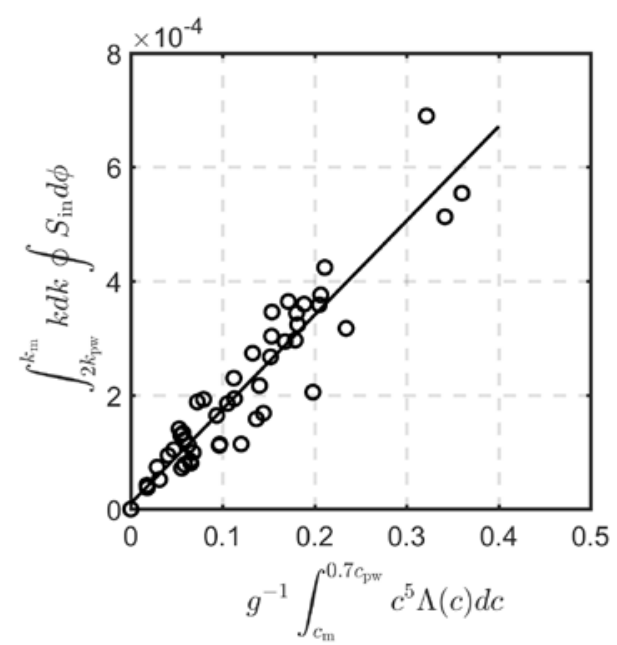

F i g. 8. Wave energy dissipation velocity as compared to the wind energy inflow (solid line shows data approximation by linear dependence)

\section{Discussion of the results}

Using our in situ data on wind velocity, spectra of wind waves and breaking characteristics, we compare the values of $b$ calculated by formula (7) with the parameters of waves and atmosphere.

In Fig. 9 the dependence of $b$ on the dynamic velocity in the air and wave age is given. As can be from Fig. 9, an explicit dependence of the coefficient $b$ on $u_{*}$ and $\alpha$, according to our data, is not observed.
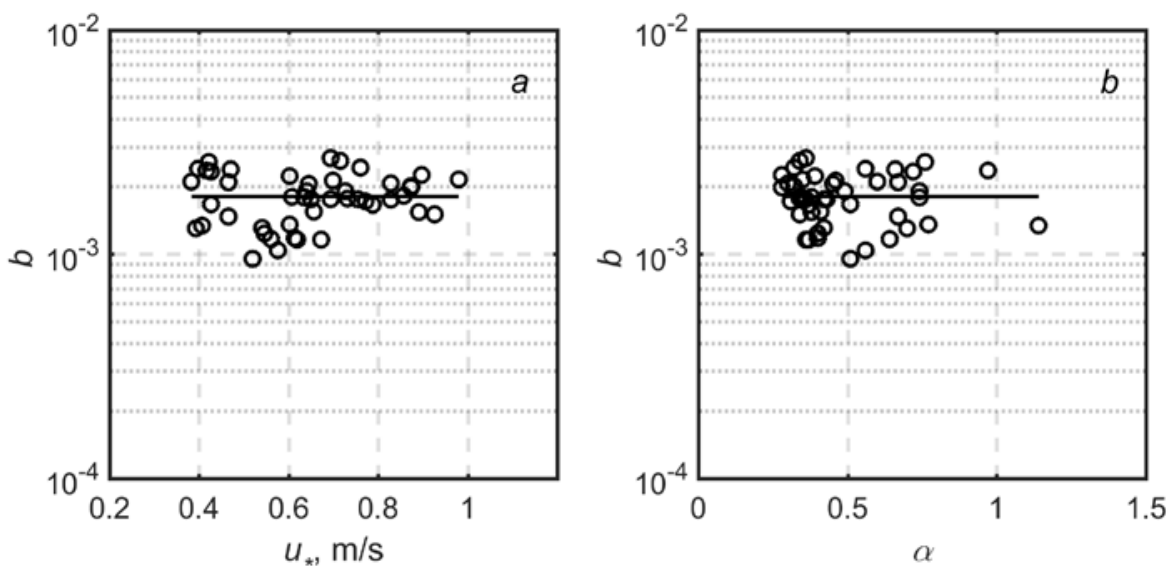

F i g. 9. Dependence of the Duncan constant on $u_{*}$ and $\alpha$ (straight line $-b=1,8 \cdot 10^{-3}$ )

An analysis of the field estimates of the coefficient $b$ value available in the literature and an explanation of the difference between them are presented in [18]. According to [18], $b$ linearly depends on the significant wave steepness $H_{\mathrm{S}} k_{\mathrm{p}} / 2$ and wave age, calculated in terms of $u_{*}: \alpha_{*}=c_{\mathrm{p}} / u_{*}$. 
The dependences of the coefficient $b$, calculated according to expression (7), on the wave parameters, as well as the data borrowed from [18, p. 2056-2057] (see Fig. 2 and 3, a), are given in Fig. 10. According to the model from [18], the coefficient $b$ decreases with the wave age increase, and rises with $H_{\mathrm{S}} k_{\mathrm{p}} / 2$ increase. In general, as follows from Fig. 10, our $b$ values correspond in order of magnitude to the values presented in $[17,18]$. At the same time, according to our measurements, no clear statistical dependence of Duncan's constant on $u_{*}, \alpha, \alpha_{*}, H_{\mathrm{S}} k_{\mathrm{p}} / 2$ was found.
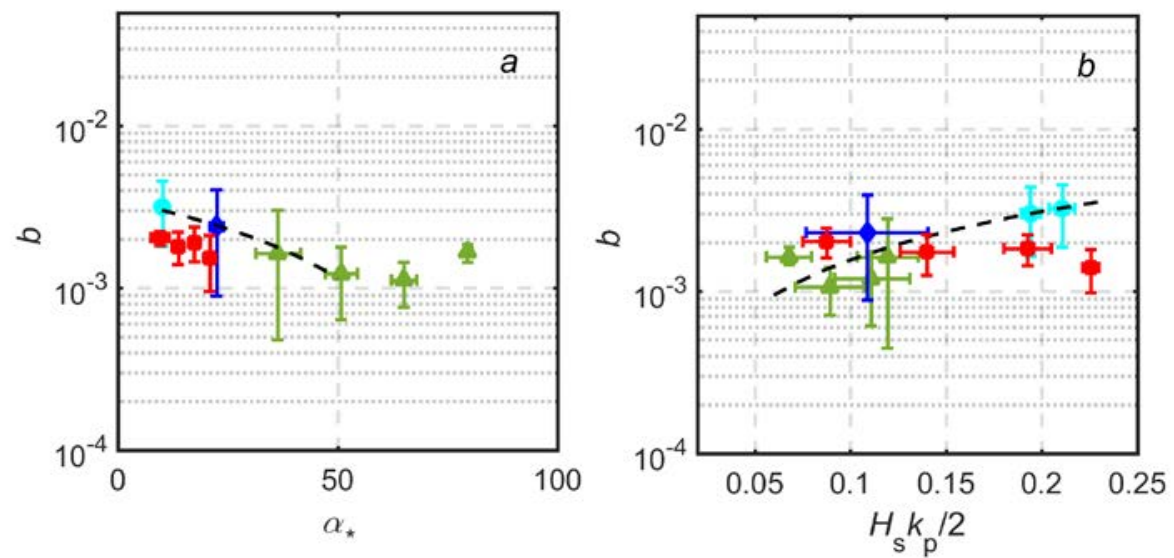

F i g. 10. Dependence of the coefficient $b$ on the waves' age (a); significant wave steepness $(b)$ ( - - based on our data; $\bullet, \mathbf{\Lambda},-$ based on the data from (see Fig. 2 and 3, a [18, p. 2056-2057]); dash line - model calculations from [18]; vertical and horizontal sections denote mean square deviations)

The applicability of $b$ and $\bar{\gamma}$ calculation results presented above will be checked by comparing some of the breaking characteristics, obtained during field measurements, with their model calculations.

In [32], the concept of an equilibrium interval [9] was applied to estimate the number of breakings per unit surface obtained in natural conditions. Taking this approach into account, the function $\Lambda(\mathbf{k})$ was expressed in terms of the wind-wave interaction coefficient $\beta(k, \phi)$ and the wave spectrum $F(\mathbf{k})$ as $\Lambda(\mathbf{k})=b^{-1} k^{3} \beta(k, \phi) F(\mathbf{k})$. Thus, a model for the number of breakings per unit surface $n_{\mathrm{br}}(\mathbf{k})=(2 \pi \bar{\gamma})^{-1} k \Lambda(\mathbf{k})$ was built:

$$
n_{\mathrm{br}}(\mathbf{k})=(2 \pi \bar{\gamma})^{-1} b^{-1} \beta(k, \phi) k^{4} F(\mathbf{k}) .
$$

Since the values of the constants $b$ and $\bar{\gamma}$ were not estimated in [32], only the form of the model dependence (8) was compared with the results of field measurements. It is shown that the slopes of the breaking velocity distributions (at $c>2.5 \mathrm{~m} / \mathrm{s}$ ) obtained from measurements and as a result of model calculations, coincide. The degree indicators of the wind dependence of the breakings total number per unit area also coincide. 
Using the results of this work (the measured wave spectrum, the values $b$ and $\bar{\gamma}$ constants), we will quantitatively compare the total number of wave breakings in the equilibrium spectral range $n_{\mathrm{br}}\left(2 k_{\mathrm{pw}}, k_{\mathrm{m}}\right)$ with experimental data.

In Fig. 11 a comparison of the model values $n_{\mathrm{br}}\left(2 k_{\mathrm{pw}}, k_{\mathrm{m}}\right)$ calculated by formula (8) and the values $n_{\mathrm{br}}^{\exp }\left(2 k_{\mathrm{pw}}, k_{\mathrm{m}}\right)$ obtained experimentally is given. The dashed straight line has a slope equal to 1 , i.e. it corresponds to the equality of the two values.

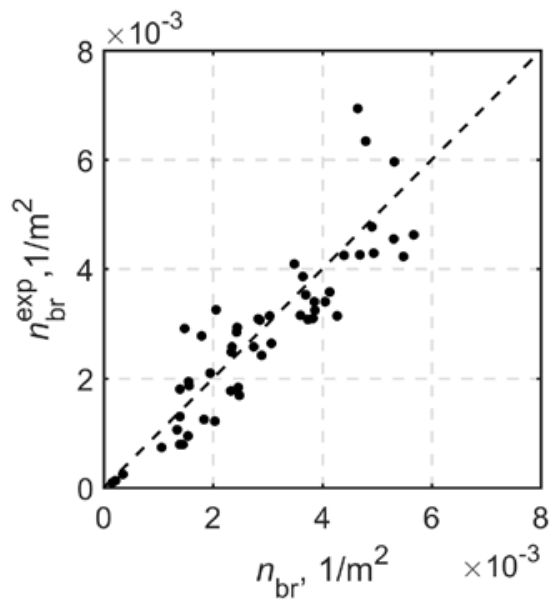

F i g. 11. Comparison of the simulated total quantity of wave breakings with the data obtained in the field conditions (dotted line corresponds to equality of two values)

As follows from Fig. 11, a good agreement between the full-scale and model values of the total number of wind wave breakings per unit area is observed.

Thus, the calculated values of Duncan's constant $b=1,8 \cdot 10^{-3}$ and $\bar{\gamma}=0,1$ make it possible to satisfactorily describe the quantitative characteristics of breaking by model functions.

\section{Conclusion}

The paper presents the results of in situ studies of geometric and dynamic characteristics of wind wave breakings. The experiments were carried out from a stationary oceanographic platform located in the Golubaya Bay near Katsiveli (Crimean Southern Coast) within the range of wind velocities from 9.2 to $21.4 \mathrm{~m} / \mathrm{s}$ with wave ages from 0.2 to 1.2 .

Determination of breaking geometric dimensions in the active phase, the velocities and directions of their movement was carried out on the basis of video surveys of the sea surface. As a result of processing of 55 video recordings, distributions of wave breaking lengths $\Lambda(c)$ were constructed. The data analysis shows that $\Lambda(c)$ have a functional dependence on $u_{*}$ and $c$ predicted by O.M. Phillips theory [9], while $\Lambda(c)$ dependence on the age of the waves is not clearly manifested. 
The probability densities of the maximum breaking length relation to the breaking wavelength calculated for different wind and wave conditions are similar and have a small scatter. The average value of $\overline{L / \lambda}$ relations obtained for all measurements is $\bar{\gamma}=0,1$.

In the present work the estimates of coefficient $b$, which relates the energy dissipation, caused by breakings, with $\Lambda(c)$ by relation (1), were carried out within the framework of the concept of the wind wave spectrum equilibrium interval. According to calculations based on our data, the average value of parameter $b$ is $1,8 \cdot 10^{-3}$. The value of $b$ we obtained is close to the results of field studies $[17,18]$. The data analysis did not reveal a clear relationship between $b$ and the parameters of waves (root-mean-square slope, wave age and the maximum steepness) and atmosphere (dynamic air velocity).

The use of the experimental estimate of parameter $b$ and $\bar{\gamma}$ obtained here provided quantitative interpretation of the measurements of the number of wave breaking per unit surface given in [32].

\section{REFERENCES}

1. Kudryavtsev, V.N., Shrira, V., Dulov, V.A. and Malinovsky, V.V., 2008. On the Vertical Structure of Wind-Driven Sea Currents. Journal of Physical Oceanography, 38(10), pp. 21212144. doi:10.1175/2008JPO3883.1

2. Zappa, C.J., McGillis, W.R., Raymond, P.A., Edson, J.B., Hintsa, E.J., Zemmelink, H.J., Dacey, J.W.H. and Ho, D.T., 2007. Environmental Turbulent Mixing Controls on Air-Water Gas Exchange in Marine and Aquatic Systems. Journal of Geophysical Research Letters, 34(10), L10601. doi:10.1029/2006GL028790

3. Thorpe, S.A., 1993. Energy Loss by Breaking Waves. Journal of Physical Oceanography, 23(11), pp. 2498-2502. doi:10.1175/1520-0485(1993)023<2498:ELBBW>2.0.CO;2

4. Melville, W.K. and Matusov, P., 2002. Distribution of Breaking Waves at the Ocean Surface. Nature, 417(6884), pp. 58-63. doi:10.1038/417058a

5. Gemmrich, J.R., Banner, M.L. and Garrett, C., 2008. Spectrally Resolved Energy Dissipation Rate and Momentum Flux of Breaking Waves. Journal of Physical Oceanography, 38(6), pp. 1296-1312. doi:10.1175/2007JPO3762.1

6. Kleiss, J.M. and Melville, W.K., 2010. Observations of Wave Breaking Kinematics in FetchLimited Seas. Journal of Physical Oceanography, 40(12), pp. 2575-2604. doi:10.1175/2010JPO4383.1

7. Kleiss, J.M. and Melville, W.K., 2011. The Analysis of Sea Surface Imagery for Whitecap Kinematics. Journal of Atmospheric and Oceanic Technology, 28(2), pp. 219-243. doi:10.1175/2010JTECHO744.1

8. Mironov, A.S. and Dulov, V.A., 2008. Detection of Wave Breaking Using Sea Surface Video Records. Measurement Science and Technology, 19(1), 015405. doi:10.1088/09570233/19/1/015405

9. Phillips, O.M., 1985. Spectral and Statistical Properties of the Equilibrium Range in WindGenerated Gravity Waves. Journal of Fluid Mechanics, 156, pp. 505-531. doi:10.1017/S0022112085002221

10. Phillips, O.M., Posner, F.L. and Hansen, J.P., 2001. High Range Resolution Radar Measurements of the Speed Distribution of Breaking Events in Wind-Generated Ocean Waves: Surface Impulse and wave Energy Dissipation Rates. Journal of Physical Oceanography, 31(2), pp. 450-460. doi:10.1175/1520-0485(2001)031<0450:HRRRMO>2.0.CO;2 
11. Thomson, J., Gemmrich, J.R. and Jessup, A.T., 2009. Energy Dissipation and the Spectral Distribution of Whitecaps. Geophysical Research Letters, 36(11), L11601. doi:10.1029/2009GL038201

12. Melville, W.K., 1994. Energy Dissipation by Breaking Waves. Journal of Physical Oceanography, 24(10), $\quad$ pp. 2041-2049. doi:10.1175/15200485(1994)024<2041:EDBBW>2.0.CO;2

13. Banner, M.L. and Peirson, W.L., 2007. Wave Breaking Onset and Strength for TwoDimensional Deep-Water Wave Groups. Journal of Fluid Mechanics, 585, pp. 93-115. doi:10.1017/S0022112007006568

14. Drazen, D.A., Melville, W.K. and Lenain, L., 2008. Inertial Scaling of Dissipation in Unsteady Breaking Waves. Journal of Fluid Mechanics, 611, pp. 307-332. doi:10.1017/S0022112008002826

15. Romero, L., Melville, W.K. and Kleiss, J.M., 2012. Spectral Energy Dissipation due to Surface Wave Breaking. Journal of Physical Oceanography, 42(9), pp. 1421-1444. doi:10.1175/JPO-D-11-072.1

16. Gemmrich, J.R., Zappa, C.J., Banner, M.L. and Morison, R.P., 2013. Wave Breaking in Developing and Mature Seas. Journal of Geophysical Research: Oceans, 118(9), pp. 45424552. doi:10.1002/jgrc.20334

17. Schwendeman, M., Thomson, J. and Gemmrich, J.R., 2014. Wave Breaking Dissipation in a Young Wind Sea. Journal of Physical Oceanography, 44(1), pp. 104-127. doi:10.1175/JPOD-12-0237.1

18. Zappa, C.J., Banner, M.L., Morison, R.P. and Brumer, S.E., 2016. On the Variation of the Effective Breaking Strength in Oceanic Sea States. Journal of Physical Oceanography, 46(7), pp. 2049-2061. doi:10.1175/JPO-D-15-0227.1

19. Fairall, C.W., Bradley, E.F., Hare, J.E., Grachev, A.A. and Edson, J.B., 2003. Bulk Parameterization of Air-Sea fluxes: Updates and Verification for the COARE Algorithm. Journal of Climate, 16(4), pp. 571-591. doi:10.1175/15200442(2003)016<0571:BPOASF>2.0.CO;2

20. Large, W.G. and Pond, S., 1982. Sensible and Latent Heat flux Measurements over the Ocean. Journal of Physical Oceanography, 12(5), pp. 464-482. doi:10.1175/15200485(1982)012<0464:SALHFM>2.0.CO;2

21. Phillips, O.M., 1958. The Equilibrium Range in the Spectrum of Wind-Generated Waves. Journal of Fluid Mechanics, 4(4), pp. 426-434. doi:10.1017/S0022112058000550

22. Hanson, J.L. and Phillips, O.M., 1999. Wind Sea Growth and Dissipation in the Open Ocean. Journal of Physical Oceanography, 29(8), pp. 1633-1648. doi:10.1175/15200485(1999)029<1633:WSGADI>2.0.CO;2

23. Callaghan, A.H., Deane, G.B., Stokes, M.D. and Ward, B., 2012. Observed Variation in the Decay Time of Oceanic Whitecap Foam. Journal of Geophysical Research: Oceans, 117(C9), C09015. doi:10.1029/2012JC008147

24. Rapp, R.J. and Melville, W.K., 1990. Laboratory Measurements of Deep-Water Breaking Waves. Philosophical Transactions of the Royal Society of London. Series A, Mathematical and Physical Sciences, 331(1622), pp. 735-800. doi:10.1098/rsta.1990.0098

25. Stansell, P. and MacFarlane, C., 2002. Experimental Investigation of Wave Breaking Criteria Based on Wave Phase Speeds. Journal of Physical Oceanography, 32(5), pp. 1269-1283. doi:10.1175/1520-0485(2002)032<1269:EIOWBC>2.0.CO;2

26. Duncan, J.H., 1981. An Experimental Investigation of Breaking Waves Produced by a Towed Hydrofoil. Proceedings of the Royal Society A, 377(1770), pp. 331-348. doi:10.1098/rspa.1981.0127

27. Jessup, A.T. and Phadnis, K.R., 2005. Measurement of the Geometric and Kinematic Properties of Microscale Breaking Waves from Infrared Imagery Using a PIV Algorithm. Measurement Science and Technology, 16(10), pp. 1961-1969. doi:10.1088/09570233/16/10/011 
28. Sutherland, P. and Melville, W.K., 2013. Field Measurements and Scaling of Ocean Surface Wave-Breaking Statistics. Geophysical Research Letters, 40(12), pp. 3074-3079. doi:10.1002/grl.50584

29. Thomson, J., D'Asaro, E.A., Cronin, M.F., Rogers, W.E., Harcourt, R.R., and Shcherbina, A., 2013. Waves and the Equilibrium range at Ocean Weather Station P. Journal of Geophysical Research: Oceans, 118(11), pp. 5951-5962. doi:10.1002/2013JC008837

30. Plant, W.J., 1982. A Relation between Wind Stress and Wave Slope. Journal of Geophysical Research: Oceans, 87(C3), pp. 1961-1967. doi:10.1029/JC087iC03p01961

31. Meirink, J.F., Makin, V.K. and Kudryavtsev, V.N., 2003. Note on the Growth Rate of Water Waves Propagating at an Arbitrary Angle to the Wind. Boundary-Layer Meteorology, 106(1), pp. 171-183. doi:10.1023/A:1020835211837

32. Korinenko, A.E., Malinovsky, V.V. and Kudryavtsev, V.N., 2018. Experimental Research of Statistical Characteristics of Wind Wave Breaking. Physical Oceanography, 25(6), pp. 489-500. doi:10.22449/1573-160X-2018-6-489-500

About the authors:

Aleksandr E. Korinenko, Research Associate, Marine Hydrophysical Institute of RAS (2 Kapitanskaya St., Sevastopol, 299011, Russian Federation), Ph. D. (Phys.-Math.), Scopus Author ID: 23492523000, korinenko.alex@gmail.com

Vladimir V. Malinovsky, Senior Research Associate, Marine Hydrophysical Institute of RAS (2 Kapitanskaya St., Sevastopol, 299011, Russian Federation), Ph. D. (Phys.-Math.), ORCID ID: 0000-0002-5799-454X, ResearcherID: F-8709-2014, SPIN-code: 9206-3020, Scopus Author ID: 23012976200, vladimir.malinovsky@mhi-ras.ru

Vladimir N. Kudryavtsev, Executive Director of the Laboratory of Satellite Oceanography, Russian State Hydrometeorological University (79 Voronezhskaya St., Saint Petersburg, 192007, Russian Federation); Leading Research Associate, Marine Hydrophysical Institute of RAS (2 Kapitanskaya St., Sevastopol, 299011, Russian Federation), Dr. Sci. (Phys.-Math.), Scopus Author ID: 7102703183, kudr@rshu.ru

Vladimir A. Dulov, Executive Director of Applied Sea Physics Laboratory, Marine Hydrophysical Institute of RAS (2 Kapitanskaya St., Sevastopol, 299011, Russian Federation), Dr. Sci. (Phys.-Math.), ORCID ID: 0000-0002-0038-7255, ResearcherID: F-8868-2014, SPIN-code: 8303-6244, Scopus Author ID: 6602725409, dulov@mhi-ras.ru

Contribution of the co-authors:

Aleksandr E. Korinenko - development of techniques and carrying out the experimental studies, analysis and synthesis of the research results, preparation of the text of the paper

Vladimir V. Malinovsky - development of experimental research method, analysis and synthesis of research results, participation in the discussion of the article materials, preparation of the paper text

Vladimir N. Kudryavtsev - general scientific management of the research, description of the research results, refinement of the text of the paper

Vladimir A. Dulov - development of experimental research method, participation in the discussion of the article materials, preparation of the paper text

All the authors have read and approved the final manuscript.

The authors declare that they have no conflict of interest. 